\title{
Eltrombopag before Procedures in Patients with Cirrhosis and Thrombocytopenia
}

\author{
Nezam H. Afdhal, M.D., Edoardo G. Giannini, M.D., Ph.D., Ghias Tayyab, M.D., \\ Aftab Mohsin, M.D., Jin-Woo Lee, M.D., Ph.D., Angelo Andriulli, M.D., \\ Lennox Jeffers, M.D., John McHutchison, M.D., Pei-Jer Chen, M.D., Ph.D., \\ Kwang-Hyub Han, M.D., Fiona Campbell, B.Sc., Denise Hyde, Ph.D., \\ Andres Brainsky, M.D., and Dickens Theodore, M.D., M.P.H., \\ for the ELEVATE Study Group*
}

A BSTRACT

The authors' affiliations are listed in the Appendix. Address reprint requests to Dr. Afdhal at the Division of Gastroenterology/ Liver Center, Beth Israel Deaconess Medical Center, 110 Francis St., Suite 8E, Boston, MA 02215, or at nafdhal@bidmc.harvard .edu.

*Additional members of the Eltrombopag Evaluated for Its Ability to Overcome Thrombocytopenia and Enable Procedures (ELEVATE) study group are listed in the Supplementary Appendix, available at NEJM.org.

N Engl J Med 2012;367:716-24. DOI: 10.1056/NEJMoal110709

Copyright (๑) 2012 Massachusetts Medical Society.

\section{BACKGROUND}

Eltrombopag is an oral thrombopoietin-receptor agonist. This study evaluated the efficacy of eltrombopag for increasing platelet counts and reducing the need for platelet transfusions in patients with thrombocytopenia and chronic liver disease who are undergoing an elective invasive procedure.

\section{METHODS}

We randomly assigned 292 patients with chronic liver disease of diverse causes and platelet counts of less than 50,000 per cubic millimeter to receive eltrombopag, at a dose of $75 \mathrm{mg}$ daily, or placebo for 14 days before a planned elective invasive procedure that was performed within 5 days after the last dose. The primary end point was the avoidance of a platelet transfusion before, during, and up to 7 days after the procedure. A key secondary end point was the occurrence of bleeding (World Health Organization [WHO] grade 2 or higher) during this period.

\section{RESULTS}

A platelet transfusion was avoided in 104 of 145 patients who received eltrombopag $(72 \%)$ and in 28 of 147 who received placebo $(19 \%)(\mathrm{P}<0.001)$. No significant difference between the eltrombopag and placebo groups was observed in bleeding episodes of WHO grade 2 or higher, which were reported in $17 \%$ and $23 \%$ of patients, respectively. Thrombotic events of the portal venous system were observed in 6 patients who received eltrombopag, as compared with 1 who received placebo, resulting in the early termination of the study. The incidence and severity of other adverse events were similar in the eltrombopag and placebo groups.

\section{CONCLUSIONS}

Eltrombopag reduced the need for platelet transfusions in patients with chronic liver disease who were undergoing elective invasive procedures, but it was associated with an increased incidence of portal-vein thrombosis, as compared with placebo. (Funded by GlaxoSmithKline; ELEVATE ClinicalTrials.gov number, NCT00678587.) 
T HROMBOCYTOPENIA IS FREQUENTLY OBserved in patients with chronic liver disease, with studies suggesting that it occurs in up to $76 \%$ of patients with cirrhosis. ${ }^{1-3}$ The degree of thrombocytopenia is proportional to the severity of the liver disease. ${ }^{4,5}$ Thrombocytopenia increases the risk of bleeding during and after invasive procedures and may result in the cancellation or postponement of elective procedures. ${ }^{6}$ Platelet transfusions are commonly used to reduce the risk of bleeding during a procedure, but their short duration of efficacy and the risk of transfusion reactions limit their use. ${ }^{7,8}$ Furthermore, the development of antiplatelet antibodies (alloimmunization) can cause refractory thrombocytopenia in up to half of patients who receive multiple transfusions. ${ }^{9}$

Eltrombopag is an oral thrombopoietin-receptor agonist approved for use in patients with chronic immune thrombocytopenia. A phase 2 study showed that eltrombopag could increase platelet counts in patients with thrombocytopenia and hepatitis C. ${ }^{10}$ This article reports the results of the Eltrombopag Evaluated for Its Ability to Overcome Thrombocytopenia and Enable Procedures (ELEVATE) study - a double-blind, placebo-controlled trial that evaluated the ability of eltrombopag to increase platelet counts and reduce the need for platelet transfusions in patients with thrombocytopenia and chronic liver disease who were undergoing an elective invasive procedure.

\section{METHODS}

\section{PATIENTS}

Eligible patients were 18 years of age or older, had chronic liver disease and a platelet count of less than 50,000 per cubic millimeter, and in the opinion of the investigator, would require a platelet transfusion to manage the risk of bleeding before undergoing an elective invasive procedure. Additional eligibility criteria were a Child-Pugh score ${ }^{11}$ of 5 to 12 (on a scale from 5 to 15 , with higher scores indicating more severe liver disease) and a score of 6 to 24 on the Model for End-Stage Liver Disease criteria $^{12}$ (on a scale from 6 to 40 , with higher scores indicating more advanced liver disease). Patients were excluded if they were pregnant or if they had evidence of portal-vein thrombosis on abdominal imaging within 3 months after study entry (no baseline imaging was required), a history of arterial or venous thrombosis with on- going risk factors for thrombosis, any condition associated with World Health Organization (WHO) grade 3 or 4 bleeding, a history of abnormal platelet aggregation, or an active infection requiring systemic antibiotic agents.

\section{STUDY DESIGN}

The study was a double-blind, randomized, placebocontrolled, phase 3 trial conducted in 13 countries. Patients were assigned in a 1:1 ratio to a study group and were stratified according to the bleeding risk associated with the invasive procedure. Patients received either eltrombopag at a dose of $75 \mathrm{mg}$ or matched placebo once daily for 14 days (Fig. 1S in the Supplementary Appendix, available with the full text of this article at NEJM.org). Patients were assessed after 7 days of treatment (day 8) and 1 day after receiving the last dose of study medication (day 15). Invasive procedures were to be performed no more than 5 days after the patient received the final dose (days 15 to 19). On the day of the procedure, patients with a platelet count of more than 80,000 per cubic millimeter did not receive a platelet transfusion. Patients with a platelet count of less than 50,000 per cubic millimeter received a platelet transfusion before the procedure, according to local guidelines. Patients with a platelet count between 50,000 and 80,000 per cubic millimeter received a platelet transfusion at the discretion of the investigator or physician performing the procedure and were treated according to local guidelines. Assessments were performed on days 7, 14, 21, and 30 of follow-up. The study was concluded after the assessment on day 30 of follow-up.

An independent data and safety monitoring committee was established to review safety data during the study. A clinical events committee reviewed data from ophthalmic assessments.

\section{STUDY OVERSIGHT}

The study protocol, available at NEJM.org, was approved by the institutional review board or ethics committee at each participating center. The study was conducted in accordance with the protocol and the Declaration of Helsinki, Good Clinical Practice guidelines, and local laws and regulations. All patients provided written informed consent.

The sponsor, GlaxoSmithKline, and the authors designed the study, developed the protocol, and analyzed the data. All authors had full access to the data, contributed to the writing of the manu- 
script, and vouch for the completeness and accuracy of the reported data and analysis and for the fidelity of the study to the protocol. Assistance in writing the first draft of the manuscript was provided by a professional medical writer paid by the sponsor.

The primary efficacy end point was the proportion of patients who did not require a platelet transfusion before, during, and up to 7 days after the elective invasive procedure. The key secondary end point was the proportion of patients with bleeding (WHO grade 2 or higher) before, during, and up to 7 days after the procedure. Other secondary end points included the number of platelet transfusions administered before, during, and up to 30 days after the procedure, assessments of platelet counts throughout the study period, and assessments related to safety and adverse events.

\section{STATISTICAL ANALYSIS}

To estimate the sample size required for analysis of the primary end point, we assumed that the proportion of patients who would meet the primary end point would be $20 \%$ for placebo and $50 \%$ for eltrombopag (see the statistical analysis plan, included in the protocol). A total of 120 patients (60 in each study group) would provide $90 \%$ power to detect a significant treatment effect with the use of a two-sided alpha level of 0.05 . To estimate the sample size required for analysis of the key secondary end point, we assumed that the proportion of patients in the placebo group with bleeding would be $20 \% .{ }^{13}$ Noninferiority with respect to the occurrence of bleeding was inferred by ensuring that the upper confidence limit (corresponding to the two-sided $95 \%$ confidence interval) for the between-group difference (the rate of bleeding with eltrombopag minus the rate with placebo) did not exceed 10 percentage points. A total of 500 patients ( 250 in each study group) would provide $80 \%$ power to rule out this absolute difference of 10 percentage points at a one-sided alpha level of 0.025 . Therefore, the planned sample was 500 patients.

Following a recommendation from the independent data and safety monitoring committee to suspend recruitment and dosing, owing to an increased frequency of thrombotic events in the eltrombopag group, the study was terminated by GlaxoSmithKline to allow a full analysis of the safety and efficacy data. All prespecified efficacy end points were assessed on an intention-to- treat basis. Safety was assessed among all patients who underwent randomization and received at least one dose of study medication.

In subgroup analyses prespecified in the protocol, the primary end point was analyzed separately for randomization strata and subgroups defined on the basis of geographic region, sex, race, age, Model for End-Stage Liver Disease score, Child-Pugh class, and weight.

\section{RESULTS}

\section{PATIENTS}

From June 2008 through September 2009, a total of 292 patients were enrolled and randomly assigned to a study group (Fig. $1 S$ in the Supplementary Appendix). Two patients in each group withdrew from the study before treatment began. Of a total of 288 patients, 145 received placebo and 143 received eltrombopag at a dose of $75 \mathrm{mg}$ once daily. The baseline demographic and clinical characteristics of the study groups were similar (Table 1). The median age was 53 years (range, 19 to 83); 64\% of the patients were men, and $61 \%$ were white. A total of 252 patients (86\%) had cirrhosis, with $10 \%$ of all patients having Child-Pugh class $\mathrm{C}$ disease (i.e., a score of 10 or higher) at baseline. The cause of chronic liver disease was viral in most cases (80\%).

A majority of patients in both study groups (62\% in the eltrombopag group and 56\% in the placebo group) underwent elective invasive procedures that were in the lowest bleeding-risk category (Table $1 \mathrm{~S}$ in the Supplementary Appendix). Overall, 20 patients receiving placebo and 14 receiving eltrombopag did not undergo an elective invasive procedure during the study.

\section{EFFICACY}

Treatment with eltrombopag increased platelet counts in the majority of patients, whereas the counts remained essentially unchanged in the placebo group (Fig. 1A). On day 1, 94\% of patients receiving placebo and $92 \%$ of those receiving eltrombopag had platelet counts of less than 50,000 per cubic millimeter. On day $15,59 \%$ of patients treated with eltrombopag had a platelet count of more than 80,000 per cubic millimeter, as compared with $5 \%$ of those receiving placebo. Median platelet counts returned to values approaching baseline counts within 4 weeks after the procedure. 
The primary end point was achieved in $72 \%$ of the patients (104 of 145) in the eltrombopag group, as compared with $19 \%$ (28 of 147$)$ in the placebo group $(\mathrm{P}<0.001)$. The proportion of patients with a platelet count between 50,000 and 80,000 per cubic millimeter who did not receive a platelet transfusion was similar in the two groups $(14 \%$ in the eltrombopag group and $12 \%$ in the placebo group), confirming that the subjective decision to administer a platelet transfusion was not influenced by the group assignment and did not influence the primary analysis. Two patients in the eltrombopag group and 3 in the placebo group did not receive a platelet transfusion despite having a platelet count of less than 50,000 per cubic millimeter. The results of the primary analysis were also consistent with those of prespecified subgroup analyses (Fig. 1B), with clinically meaningful differences observed for most subgroups, including patients with advanced liver disease and those who underwent invasive procedures with the highest bleeding risk.

The quantity of platelets administered to patients during each transfusion episode was lower in patients receiving eltrombopag than in those receiving placebo. The median number of transfused platelet units was 3.0 (2.5 units from a single donor and 3.0 units from multiple donors) in the eltrombopag group, as compared with a median of 4.0 platelet units (2.0 units from a single donor and 5.0 units from multiple donors) in the placebo group.

The key secondary end point of noninferiority with regard to the rate of bleeding episodes (with a noninferiority margin of 10 percentage points) was met (23\% in the placebo group and $17 \%$ in the eltrombopag group; absolute difference, -6 percentage points; $95 \%$ confidence interval [CI], -15 to 3$)$.

\section{SAFETY}

The incidence and severity of adverse events were similar in the two groups. The most common adverse events, occurring in more than $3 \%$ of patients in either study group, are reported in Table 2. Those occurring in at least $5 \%$ of patients in either group were headache, pyrexia, abdominal pain, diarrhea, nausea, and hepatic encephalopathy. A complete list of adverse events is provided in Table $2 S$ in the Supplementary Appendix. The incidence of elevated alanine aminotransferase levels was similar in the two study groups $(0 \%$ in
Table 1. Baseline Demographic and Clinical Characteristics.*

\begin{tabular}{|c|c|c|}
\hline Characteristic & $\begin{array}{l}\text { Placebo } \\
(\mathrm{N}=147)\end{array}$ & $\begin{array}{l}\text { Eltrombopag } \\
\quad(\mathrm{N}=145)\end{array}$ \\
\hline \multicolumn{3}{|l|}{ Age $-y r$} \\
\hline Median & 54 & 52 \\
\hline Range & $19-83$ & $19-79$ \\
\hline Male sex - no. (\%) & $92(63)$ & $96(66)$ \\
\hline \multicolumn{3}{|l|}{ Race - no. (\%) } \\
\hline White & $93(63)$ & $85(59)$ \\
\hline Asian & $52(35)$ & $55(38)$ \\
\hline Other & $2(1)$ & $5(3)$ \\
\hline \multicolumn{3}{|c|}{ Platelet count $-\times 10^{-3}$ per cubic millimeter } \\
\hline Median & 40 & 40 \\
\hline Range & $8-222$ & $12-62$ \\
\hline \multicolumn{3}{|c|}{ Child-Pugh class — no./total no. (\%) } \\
\hline A & $63 / 146(43)$ & $72 / 145(50)$ \\
\hline B & $66 / 146(45)$ & $62 / 145(43)$ \\
\hline $\mathrm{C}$ & $17 / 146(12)$ & $11 / 145(8)$ \\
\hline \multicolumn{3}{|l|}{ MELD score $\mathbb{}$} \\
\hline Median & 12 & 12 \\
\hline Range & $6-25$ & $6-24$ \\
\hline \multicolumn{3}{|c|}{$\begin{array}{c}\text { Most common cause of chronic liver disease } \\
\text { — no. (\%) }\end{array}$} \\
\hline Hepatitis C & $106(72)$ & $91(63)$ \\
\hline Hepatitis B & $16(11)$ & $22(15)$ \\
\hline Alcoholic liver disease & $11(7)$ & $10(7)$ \\
\hline
\end{tabular}

* None of the characteristics differed significantly between the two study groups; the Wilcoxon two-sample test was used for continuous measures, and Fisher's exact test for categorical measures.

$\uparrow$ Seven patients in each study group had a platelet count of 50,000 per cubic millimeter or higher on day 1.

$\mp$ Child-Pugh scores ${ }^{11}$ range from 5 to 15 , with higher scores indicating more severe disease; a score of 5 or 6 indicates class A disease, 7 to 9 class B, and 10 or higher class $C$.

$\int$ Scores for the Model for End-Stage Liver Disease ${ }^{12}$ (MELD) criteria range from 6 to 40, with higher scores indicating more advanced liver disease. One patient with a MELD score of 25 was incorrectly enrolled in the study but was still included in the analyses. Data on MELD score were missing for 7 patients in the placebo group and 10 in the eltrombopag group.

I The most common causes of chronic liver disease were those occurring in more than $5 \%$ of patients. Causes were not mutually exclusive.

the placebo group and $1 \%$ in the eltrombopag group). A majority of patients who had an adverse event $(76 \%)$ did so after the treatment (more than 1 day after the last dose of study medication).

Serious adverse events occurred in 19 patients receiving eltrombopag (13\%) and in 17 receiving placebo (12\%). The most common serious adverse events in both groups (occurring in at least 2 pa- 


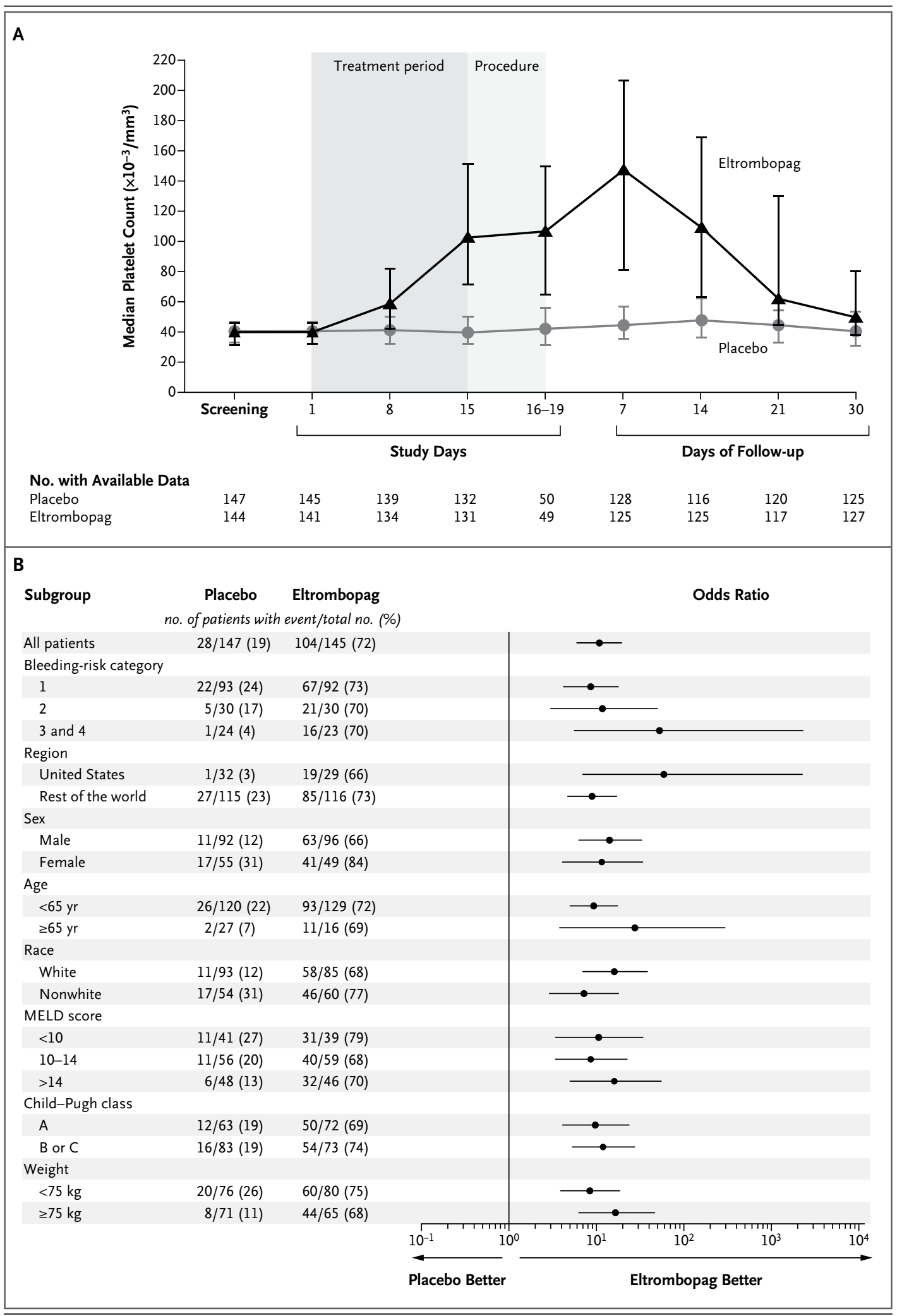


Figure 1 (facing page). Median Platelet Counts and Results of Subgroup Analyses, According to Study Group.

Panel A shows the median platelet count in patients receiving eltrombopag at a dose of $75 \mathrm{mg}$ once daily or placebo for 14 days. I bars indicate the interquartile range. Elective invasive procedures were scheduled to be performed no more than 5 days after the final dose (days 15 to 19). The maximum median platelet count (148,000 per cubic millimeter) occurred 7 days after the procedure. Panel $B$ shows the odds ratios for the avoidance of platelet transfusion before and up to 7 days after the elective procedure, according to subgroup (primary end point). The forest plot shows odds ratios for subgroups defined on the basis of demographic and baseline clinical characteristics. Bleeding-risk categories 3 and 4 (randomization strata) were combined, owing to the small samples in these categories. Scores for the Model for End-Stage Liver Disease ${ }^{12}$ (MELD) criteria range from 6 to 40, with higher scores indicating more advanced liver disease. Data on MELD score were missing for 2 patients in the placebo group and 1 in the eltrombopag group. Child-Pugh scores ${ }^{11}$ range from 5 to 15, with higher scores indicating more severe disease; a score of 5 or 6 indicates class A disease, 7 to 9 class $B$, and 10 or higher class $C$. Child-Pugh scores were missing for 1 patient in the placebo group. Horizontal bars indicate $95 \%$ confidence intervals.

tients in each group) were hepatic encephalopathy (in $2 \%$ of patients), mesenteric-vein thrombosis (in 1\%), cataracts (in 1\%), encephalopathy (in 1\%), gastroenteritis (in 1\%), rectal hemorrhage (in 1\%), and sepsis (in 1\%). With the exception of thrombotic events, rates of serious adverse events were similar in the study groups. Adverse events leading to the discontinuation of study medication occurred in 3 patients in each group. Five deaths occurred: three in the eltrombopag group and two in the placebo group. One death in the eltrombopag group (due to sepsis) was possibly related to therapy. The other two deaths in this group were due to upper gastrointestinal hemorrhage and sepsis and to lymphoma. The two deaths in the placebo group were attributed to chronic liver disease and to gastroenteritis and shock.

Eight patients had 10 thrombotic events: 6 patients ( 7 events) in the eltrombopag group and 2 patients (3 events) in the placebo group (odds ratio with eltrombopag, 3.04; 95\% CI, 0.62 to 14.82) (Table 3). Nine of the 10 events involved the portal venous system, including all the events

\begin{tabular}{lcc|}
$\begin{array}{l}\text { Table 2. Adverse Events Occurring in at Least } 3 \% \text { of Patients in Either Study } \\
\text { Group.* }\end{array}$ & Placebo & Eltrombopag \\
Event & (N=145) & (N 143$)$ \\
& no. of patients & with event (\%) \\
& $85(59)$ & $79(55)$ \\
Any & $6(4)$ & $11(8)$ \\
Headache & $10(7)$ & $8(6)$ \\
Pyrexia & $7(5)$ & $7(5)$ \\
Abdominal pain & $5(3)$ & $7(5)$ \\
Diarrhea & $7(5)$ & $7(5)$ \\
Nausea & $3(2)$ & $6(4)$ \\
International normalized ratio increased & $1(1)$ & $5(3)$ \\
Anemia & $3(2)$ & $5(3)$ \\
Epistaxis & $3(2)$ & $5(3)$ \\
Peripheral edema & $1(1)$ & $5(3)$ \\
Pyuria & $4(3)$ & $4(3)$ \\
Ascites & $1(1)$ & $6(4)$ \\
Portal-vein or splanchnic-vein thrombosis & $3(2)$ & $4(3)$ \\
Vomiting & $5(3)$ & $3(2)$ \\
Cataract & $7(5)$ & $4(3)$ \\
Hepatic encephalopathy & $5(3)$ & $2(1)$ \\
Upper abdominal pain & $5(3)$ & $2(1)$ \\
Urinary tract infection & $4(3)$ & $1(1)$ \\
Hematuria & $4(3)$ & 0 \\
Dizziness & & \\
\hline
\end{tabular}

* Patients could have more than one adverse event.

in patients who received eltrombopag; these patients presented with symptomatic portal-vein or splanchnic-vein thromboses. The remaining patient, who received placebo, had a myocardial infarction. Of the 6 patients in the eltrombopag group who had a portal-vein thrombosis, 5 had the event when the platelet count was higher than 200,000 per cubic millimeter (the maximum platelet count achieved during the study). An association between platelet counts of 200,000 per cubic millimeter or higher and an increased risk of thrombotic events was identified in a post hoc analysis. The median time to onset after the first dose of study medication was 21.5 days (range, 15 to 53). None of the events occurred during therapy; the median time to onset after the last dose was 8.5 days (range, 1 to 38). Five of the 


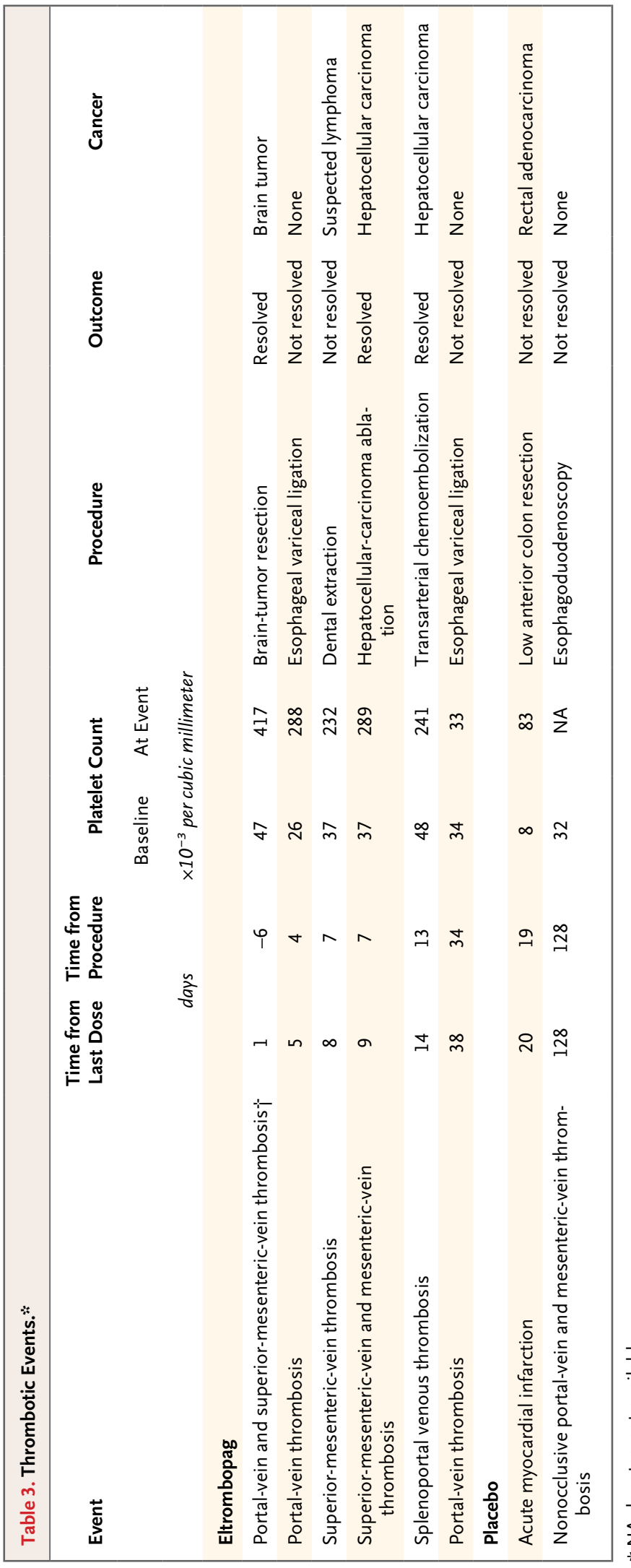

6 patients treated with eltrombopag who had a thrombotic event had the event within 2 weeks after the last dose of study medication. None of the 8 patients who had thrombotic events had received both eltrombopag and a platelet transfusion or had had any blood-product use recorded. In addition, none of the 26 patients who received both eltrombopag and a platelet transfusion had a thrombotic event. Overall, 5 of the 8 patients with a thrombotic event had evidence of cancer.

\section{DISCUSSION}

Thrombocytopenia frequently complicates the treatment of patients with chronic liver disease who require an invasive procedure as part of their routine clinical care. This study showed that a $75-\mathrm{mg}$ dose of eltrombopag administered once daily for 14 days raised platelet counts and significantly reduced the proportion of patients requiring a platelet transfusion related to an invasive procedure - findings that were consistent across important subgroups such as those defined by sex, race, age, and randomization stratum (bleeding risk associated with the procedure). Analyses of secondary efficacy end points showed that fewer platelet units were transfused in the eltrombopag group than in the placebo group and that the results in the eltrombopag group were noninferior to those in the placebo group with regard to bleeding.

Perhaps a cardinal clinical question to ask is whether there is a need for a plateletstimulating growth factor or even platelet transfusions in patients with liver disease who undergo invasive procedures. In a recent study examining the bleeding risk among patients with severe thrombocytopenia (platelet count, $<75,000$ per cubic millimeter), Giannini et al. found that $31 \%$ of the patients had bleeding. ${ }^{14}$ Interestingly, in these patients awaiting a liver transplant, the platelet count rather than coagulopathy was the major determinant of the bleeding risk. In our study, $23 \%$ of the patients who received placebo and a platelet transfusion had bleeding of WHO grade 2 or higher. Our study also showed the variation in global 
practice with regard to the use of platelet transfusions for thrombocytopenia, since a substantial number of patients with a platelet count between 50,000 and 80,000 per cubic millimeter, and even five patients with a platelet count of less than 50,000 per cubic millimeter, did not receive transfusions. Since there are no globally accepted clinical practice guidelines for platelet transfusion in patients with chronic liver disease who undergo invasive procedures, we did allow flexibility in using platelet transfusions for patients with a platelet count between 50,000 and 80,000 per cubic millimeter, a level at which the risk of bleeding is still unclear.

Doppler ultrasonography of the abdomen was not required during the study screening period, so it is possible that some of the patients at study entry had a subclinical partial portal-vein thrombosis or a low flow state, which may have contributed to the development or persistence of thromboses during the study. We considered a number of hypotheses to help explain the increased risk of portal-vein thrombosis among patients who received eltrombopag. Patients with advanced liver disease, such as cirrhosis, typically have one or more risk factors that predispose them to thrombotic events, including intraabdominal cancers, imbalances of the coagulation system, and portal hypertension with reduced portal-vein flow. ${ }^{15-17}$ Furthermore, other studies have suggested that patients who undergo an invasive procedure may be at increased risk for portal-vein thromboses, ${ }^{18}$ which have been reported in up to $35 \%$ of patients with cirrhosis and hepatocellular carcinoma. ${ }^{19}$

We hypothesized that the combination of a sustained increase in the platelet count and an associated degree of predisposing injury from the procedure could have contributed to the development of thromboses. Patients who received eltrombopag had a sustained increase in the platelet count for approximately 2 weeks after the cessation of eltrombopag treatment, whereas patients who received placebo and a platelet transfusion did not have a sustained increase in the platelet count. Thus, an undiagnosed nonocclusive portalvein thrombosis could have acted as a nidus of clotting activity for the relatively large pool of available platelets generated by eltrombopag. If there was also local inflammation or vascular endothelial injury, the risk of thrombosis would be even higher. Interestingly, four of the six pa- tients receiving eltrombopag who had a thrombus underwent procedures that involved the splanchnic circulation, such as radiofrequency ablation, transarterial chemoembolization, or variceal ligation.

Another post hoc analysis identified an association between the maximum platelet counts measured in the study and thrombotic events: patients who had a platelet count of 200,000 per cubic millimeter or higher were at increased risk for thrombotic events. This association potentially allows for the development of strategies to mitigate the risk of thrombotic events in this population. Changes in dosing (a decreased dose, less-frequent dosing, or a shorter duration of dosing) could be used to minimize the proportion of patients who have a platelet count of 200,000 per cubic millimeter or higher, while maintaining a platelet count that is sufficiently high for the patient to undergo an elective invasive procedure without having substantial bleeding or requiring a platelet transfusion.

In conclusion, treatment with eltrombopag at a dose of $75 \mathrm{mg}$ once daily for 14 days reduced the need for platelet transfusions in patients with chronic liver disease and thrombocytopenia who were undergoing elective invasive procedures. Platelet counts were increased during treatment with eltrombopag and for up to 2 weeks after treatment. However, an increased risk of portal-vein thrombosis was observed among patients receiving eltrombopag. Further exploration of eltrombopag therapy is required, including better identification of risk factors for the development of thrombosis, dose optimization, and careful patient selection. Until such studies have been conducted, eltrombopag is not recommended as an alternative to platelet transfusion in patients with chronic liver disease and thrombocytopenia who are undergoing an elective invasive procedure.

Supported by GlaxoSmithKline.

Dr. Afdhal reports receiving consulting fees from Vertex Pharmaceuticals, Merck, Pharmasset, Boehringer Ingelheim, Idera Pharmaceuticals, GlaxoSmithKline, Biolex Therapeutics, FibroGen, Human Genome Sciences, Quest Diagnostics, and Novartis; receiving grant support from Vertex Pharmaceuticals, GlaxoSmithKline, Merck, Gilead Sciences, Pharmasset, and Abbott Laboratories; receiving stock options from Medgenics and Spring Bank Pharmaceuticals; receiving travel reimbursement from GlaxoSmithKline and Merck; and being a member of the board for Idenix Pharmaceuticals, Gilead Sciences, Merck, Medgenics, Spring Bank Pharmaceuticals, and Ligand; Dr. Giannini, being a member of the board and receiving grant support, consulting fees, lecture fees, and travel reimbursement from GlaxoSmithKline and participating as an expert witness at a Strategic Advisory 
Group meeting at the European Medicines Agency concerning eltrombopag for GlaxoSmithKline; Dr. McHutchison, being an employee of and owning stock in Gilead Sciences; Dr. Chen, receiving grant support and lecture fees from Bristol-Myers Squibb; receiving travel reimbursement from Bristol-Myers Squibb and Bayer; and being a member of the board for Bristol-Myers Squibb, Bayer, GlaxoSmithKline, and Roche; Dr. Han, receiving grant support from GlaxoSmithKline; Dr. Hyde, receiving travel reimbursement from GlaxoSmithKline and being an employee of and owning stock in GlaxoSmithKline; Ms. Campbell, being an employee of and owning stock in GlaxoSmithKline and receiving travel reimbursement from GlaxoSmithKline; Dr. Brainsky, being an employee of and owning stock in GlaxoSmithKline and holding two pending patents that relate to eltrombopag; and Dr. Theodore, being an employee of and owning stock in GlaxoSmithKline, and being the co-inventor on two eltrombopag patents held by GlaxoSmithKline. No other conflicts relevant to this article were reported.
Disclosure forms provided by the authors are available with the full text of this article at NEJM.org.

We thank the study coordinators, nurses, and patients involved in the study; Andrew Street (MediTech Media) for assistance in writing the first draft of the manuscript; the following GlaxoSmithKline employees: Alison Moorat, Ronny Odegbami, Souria Dougherty, and all the study monitors and regional staff for contributing to the conduct of the study; Yasser Mostafa Mostafa Kamel for medical monitoring; Julian Jenkins, Michael Arning, Ann Phillips (a former employee of GlaxoSmithKline), Rich Francovitch, and Rita Patwardhan for contributing to the study design and interpretation of the data; Nicole Blackman for contributing to the study design and statistical analysis; Stephanie Qiu for statistical programming; Tobin Faulkner (a former employee of GlaxoSmithKline) for data management; and Kathleen Cuca and Manuel Aivado for review of an earlier version of the manuscript.

\section{APPENDIX}

The authors' affiliations are as follows: the Division of Gastroenterology/Liver Center, Beth Israel Deaconess Medical Center, Boston (N.H.A.); the Gastroenterology Unit, Department of Internal Medicine, University of Genoa, Genoa (E.G.G.), and the Department of Internal Medicine, Division of Gastroenterology, Casa Sollievo Sofferenza Hospital, San Giovanni Rotondo (A.A.) - both in Italy; the Department of Medicine, Gastroenterology, and Hepatology, Postgraduate Medical Institute, and Lahore General Hospital (G.T.), and the Department of Gastroenterology, Services Hospital Lahore, Services Institute of Medical Sciences (A.M.) - both in Lahore, Pakistan; the Division of Hepatology, Department of Internal Medicine, Inha University School of Medicine, Incheon (J.-W.L.), and the Department of Internal Medicine, Yonsei Liver Cancer Special Clinic, Yonsei Institute of Gastroenterology, Yonsei University College of Medicine, Seoul (K.-H.H.) - both in South Korea; the Center for Liver Diseases, University of Miami, Miller School of Medicine, Miami (L.J.); Duke Clinical Research Institute and Division of Gastroenterology, Duke University Medical Center, Durham, NC (J.M.); the Department of Internal Medicine, Graduate Institute of Clinical Medicine, Hepatitis Research Center, National Taiwan University College of Medicine and National Taiwan University Hospital, Taipei City, Taiwan (P.-J.C.); and Oncology Clinical Development, GlaxoSmithKline - in Stockley Park, Uxbridge, United Kingdom (F.C., D.H.), Collegeville, PA (A.B.), and Research Triangle Park, NC (D.T.)

REFERENCES

1. Qamar AA, Grace ND, Groszmann RJ, et al. Incidence, prevalence, and clinical significance of abnormal hematologic indices in compensated cirrhosis. Clin Gastroenterol Hepatol 2009;7:689-95.

2. Bashour FN, Teran JC, Mullen KD. Prevalence of peripheral blood cytopenias (hypersplenism) in patients with nonalcoholic chronic liver disease. Am J Gastroenterol 2000;95:2936-9.

3. Giannini EG. Thrombocytopenia in chronic liver disease and pharmacologic treatment options. Aliment Pharmacol Ther 2006;23:1055-65.

4. Peck-Radosavljevic M. Thrombocytopenia in liver disease. Can J Gastroenterol 2000;14:Suppl D:60D-66D.

5. Sallah S, Bobzien W. Bleeding problems in patients with liver disease: ways to manage the many hepatic effects on coagulation. Postgrad Med 1999;106:187-90, 193-5. 6. Afdhal N, McHutchison J, Brown R, et al. Thrombocytopenia associated with chronic liver disease. J Hepatol 2008;48: 1000-7.

7. Trotter JF. Coagulation abnormalities in patients who have liver disease. Clin Liver Dis 2006;10:665-78.
8. Poordad F. Thrombocytopenia in chronic liver disease. Aliment Pharmacol Ther 2007;26:Suppl 1:5-11.

9. Slichter SJ. Evidence-based platelet transfusion guidelines. Hematology Am Soc Hematol Educ Program 2007:172-8. 10. McHutchison JG, Dusheiko G, Shiffman ML, et al. Eltrombopag for thrombocytopenia in patients with cirrhosis associated with hepatitis C. $\mathrm{N}$ Engl J Med 2007;357:2227-36.

11. Pugh RN, Murray-Lyon IM, Dawson JL, Pietroni MC, Williams R. Transection of the oesophagus for bleeding oesophageal varices. Br J Surg 1973;60:646-9.

12. Kamath PS, Wiesner RH, Malinchoc $\mathrm{M}$, et al. A model to predict survival in patients with end-stage liver disease. Hepatology 2001;33:464-70.

13. Heddle NM, Cook RJ, Webert KE, Sigouin C, Rebulla P. Methodologic issues in the use of bleeding as an outcome in transfusion medicine studies. Transfusion 2003;43:742-52.

14. Giannini EG, Greco A, Marenco S, Andorno E, Valente U, Savarino V. Incidence of bleeding following invasive procedures in patients with thrombocyto- penia and advanced liver disease. Clin Gastroenterol Hepatol 2010;8:899-902. 15. Lisman T, Caldwell SH, Burroughs $\mathrm{AK}$, et al. Hemostasis and thrombosis in patients with liver disease: the ups and downs. J Hepatol 2010;53:362-71.

16. Tripodi A, Primignani M, Mannucci PM. Abnormalities of hemostasis and bleeding in chronic liver disease: the paradigm is challenged. Intern Emerg Med 2010;5:7-12.

17. Amitrano L, Guardascione MA, Ames PR. Coagulation abnormalities in cirrhotic patients with portal vein thrombosis. Clin Lab 2007;53:583-9.

18. De Stefano V, Martinelli I. Splanchnic vein thrombosis: clinical presentation, risk factors and treatment. Intern Emerg Med 2010;5:487-94.

19. Kulik LM, Carr BI, Mulcahy MF, et al. Safety and efficacy of 90Y radiotherapy for hepatocellular carcinoma with and without portal vein thrombosis. Hepatology 2008;47:71-81.

Copyright (c) 2012 Massachusetts Medical Society. 